ISSN: 2386-3919 - e-ISSN: 2386-3927

DOI: https://doi.org/10.14201/et201937285111

\title{
EL PENSAMIENTO DE LOS DOCENTES SOBRE EL USO FORMATIVO DEL TELÉFONO MÓVIL
}

\section{Teachers' perception on the formative use of mobile phone}

\author{
María Luisa SEVILlano García \\ Universidad Nacional de Educación a Distancia. UNED. España \\ Correo-e:mlsevillano@edu.uned.es \\ Cristina MAROTO DEL Olmo \\ Profesora de Secundaria. Burgos
}

Carolina Pascual Moscoso

Universidad de Oviedo

Recibido: 23/10/2019; Aceptado: 30/11/2019; Publicado: 30/12/2019

Ref. Bibl. MARÍA LUISA SEVILLANO GARCÍA, CRISTINA MAROTO DEL OLMO y CAROLINA PASCUAL MOSCOSO. El pensamiento de los docentes sobre el uso formativo del teléfono móvil. Enseñanza E Teaching, 37, 2-2019, 85-111.

RESUMEN: Este trabajo de investigación analiza la visión que tienen los docentes de Educación Secundaria Obligatoria y Formación Profesional, del uso educativo del teléfono móvil en el aula. Las instituciones educativas han de ser conscientes de que estamos ante un dispositivo que ha marcado especialmente la sociedad actual y deberían esforzarse por integrar y normalizar estas potenciales herramientas educativas. La investigación se ha planteado haciendo llegar un cuestionario a los docentes, consiguiendo una muestra de 42 respuestas. Con los resultados obtenidos, hemos realizado un estudio exploratorio y descriptivo con un enfoque cuantitativo, a través de un análisis estadístico univariado y bivariado para obtener la máxima información del cruce de variables. Los resultados muestran conocimiento, por parte de los docentes, de alguna experiencia educativa haciendo uso del teléfono móvil y además manifiestan interés en conocer cómo llevar a cabo su integración educativa en el aula, con mayor incidencia entre los docentes de menor edad, más flexibles y 
receptivos y que, a su vez, muestran un nivel más alto de conocimientos Mediante este estudio se ha pretendido continuar otro ya comenzado hace tres años, por lo que hemos comparado los resultados actuales con los obtenidos entonces, apreciando una evolución positiva en cuanto a los usos, actitudes y posibilidades educativas que ven los docentes al teléfono móvil.

Palabras clave: teléfono móvil; docentes; utilidades; metodología.

SUMMARY: This study analyses the idea which Secondary School and Vocational Education teachers have of the educational use of smartphones in the classroom. Educational institutions must be aware of the especial influence of these gadgets in our current society and should strive to integrate and normalize these potential educational tools. The research has been raised by sending a questionnaire to teachers, getting a sample of 42 answers. We carried out an exploratory and descriptive study throughout the results obtained, applying a quantitative approach with univariate and bivariate statistical analysis in order to obtain the maximum information on the crossing of variables: The results show that the teachers have knowledge about some educational experiences using the mobile phone as well as show interest in learning how to carry out their educational integration in the classroom, being more flexible and receptive teachers the younger, who also show a higher level of ICT knowledge. Through this study we have tried to continue another one already started three years ago, therefore we have compared the current results with those obtained then, appreciating a positive evolution regarding the uses, attitudes and educational possibilities that teachers perceive on the mobile phone.

Key words: mobile phone; teachers; utilities; methodology.

\section{INTRODUCCIÓN Y ESTADO DE LA CUESTIÓN}

Se estima que en 2019 el número de usuarios de internet en el mundo supera la mitad de la población global. Esto queda patente en la nueva edición del informe que presentaron We Are Social y Hootsuite a principios de 2019, sobre muestras estadísticas, análisis y principales tendencias acerca del número de usuarios de internet, uso de la telefonía móvil, redes sociales y comercio online a nivel global (González, 2019; Kemp, 2019). Se trata de la octava edición de este informe anual, que recoge datos de 230 países. Existen casi 4.400 millones de usuarios de internet en el mundo, lo que supone el 57\% de la población. El 67\% de la población mundial dispone de teléfono móvil, es decir, 5.112 millones de personas, un 2\% más que el año 2018. El resumen de las conclusiones más relevantes lo podemos ver en la siguiente imagen. 
FIGURA 1

Resumen del informe Global Digital 2019

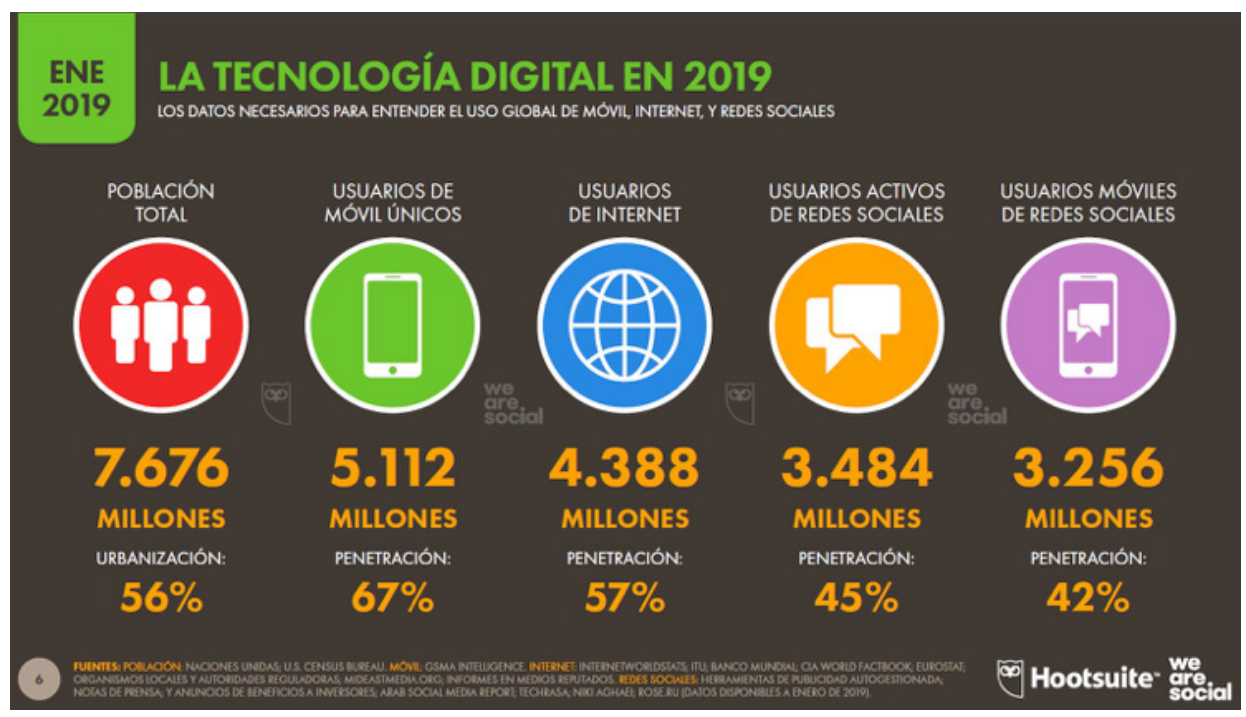

La evolución en el número de usuarios de internet en el mundo es notable, su crecimiento ha sido constante y, en la actualidad, el estudio señala que se logran captar más de un millón de usuarios nuevos cada día. Otro dato relevante es que cada vez hay más usuarios de internet que se conectan vía móvil y prefieren navegar desde sus dispositivos móviles. En 2019 el total de usuarios de internet activos vía móvil es de 3.986 millones, esto quiere decir que el 52\% de la población accede a sus contenidos favoritos y navega por medio de su smartphone (González, 2019; Kemp, 2019).

En cuanto a las cifras del informe para España, los usuarios de internet han aumentado 4 millones durante 2018, alcanzando al 93\% de la población, lo que significa que casi 43 millones de españoles acceden a la Red. En la siguiente imagen podemos ver el mismo resumen mostrado más arriba a nivel global, en este caso para España.

Otro de los datos que nos muestra el estudio, es relativo al uso que hacemos del móvil: el 85\% utiliza mensajería, el 82\% vídeos, el 75\% la navegación mediante mapas, el $51 \%$ accede a banca móvil y el $49 \%$ lo utiliza para jugar. Aunque el informe del cual hemos obtenido los datos mencionados destaca los principales usos del teléfono móvil y sabemos que dicho dispositivo ha pasado de permitir realizar y recibir llamadas, a convertirse en un dispositivo inteligente que posee múltiples opciones comunicativas, multimedia y ofimáticas: posibilidad de hacer fotos, grabar audio y vídeo, almacenar datos, música y vídeo, orientarnos por GPS, crear y editar documentos y hojas de cálculo, conectarnos vía wifi, envío de mensajería 


\section{FIGURA 2}

Resumen del informe Global Digital 2019 de España

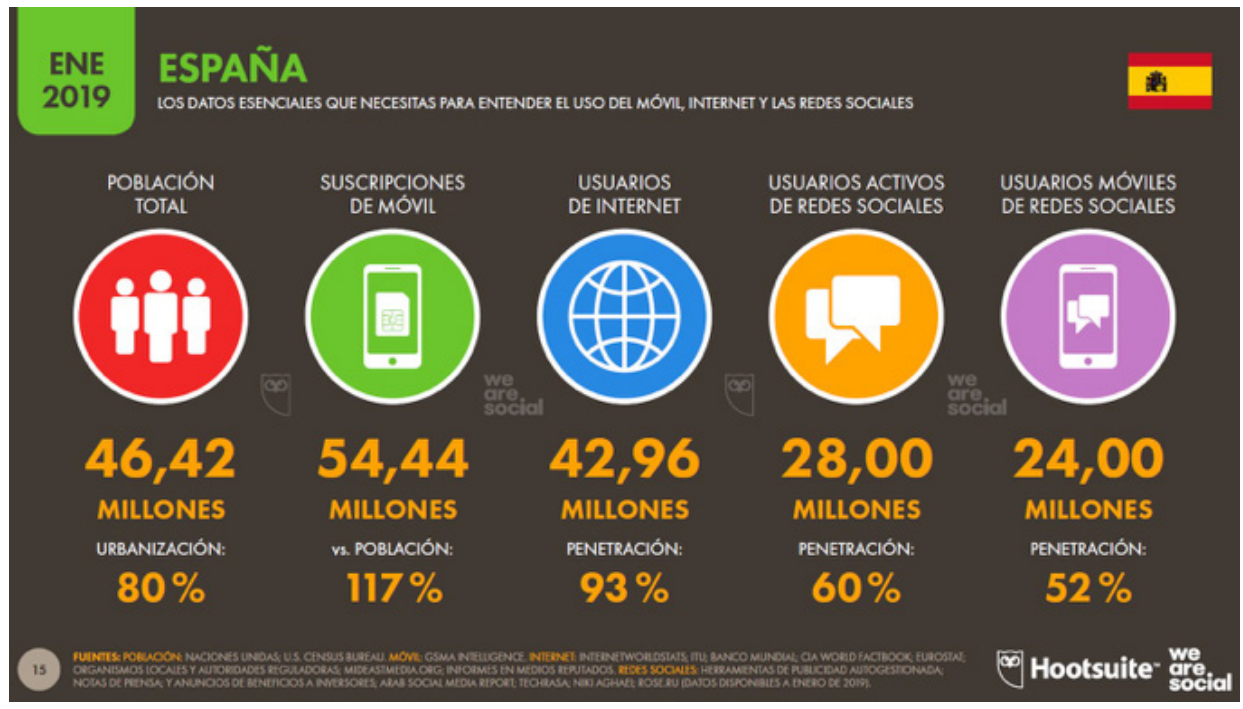

instantánea a través de apps o la navegación por internet (Brazuelo Grund, Gallego Gil y Cacheiro González, 2016). Por todo esto, el teléfono móvil adquiere ahora la categoría de smartphone o teléfono móvil inteligente con nuevos sistemas operativos a la altura de los de los ordenadores y la posibilidad de instalar aplicaciones de terceros o aplicaciones móviles (apps). Estas características, junto a su facilidad de uso, portabilidad y ubicuidad, lo han convertido en el primer medio de comunicación universal de la historia trascendiendo edad, posición social o situación geográfica (Brazuelo Grund et al., 2016).

Aclaremos el concepto de ubicuidad. Dicho término hace referencia a la integración de la informática en el entorno del individuo, haciendo posible la disponibilidad de una gran cantidad de información en cualquier momento y lugar. Por lo tanto, el aprendizaje ubicuo o $u$-learning hace referencia al desarrollo en cualquier momento o lugar a través de dispositivos móviles. Otro término asociado al aprendizaje ubicuo es el m-learning, que es «aquel que conlleva el e-learning o educación online a través de dispositivos móviles» (Vázquez-Cano y Sevillano, 2019).

Volviendo al tema de la presencia del teléfono móvil en nuestras vidas, hemos de destacar que uno de los sectores sociales que ha tenido una mayor penetración del teléfono móvil es el de los jóvenes de edades entre 16 y 24 años. El 100\% disponen de teléfono móvil y, entre sus diversos usos, se sabe que, además, cada vez son más los que lo utilizan para acceder a internet. 
Para encontrar datos sobre el uso de internet y teléfono móvil inteligente por franjas de edades en nuestro país, hemos estudiado la Encuesta sobre Equipamiento y Uso de Tecnologías de Información y Comunicación en los Hogares, TIC-H 2018 (Instituto Nacional de Estadística [INE], 2018). El 86,4\% de los hogares españoles tiene acceso a la Red, frente al 83,4\% del año anterior, y el principal tipo de conexión de banda ancha es a través de un teléfono móvil (smartphone $3 \mathrm{G} \mathrm{o} 4 \mathrm{G}$ ), presente en el $83,6 \%$ de los hogares con acceso. En dichos resultados podemos encontrar también que el uso de internet en los últimos meses es prácticamente universal (98,5\%) en los jóvenes de 16 a 24 años. A partir de ese grupo de edad va descendiendo paulatinamente conforme aumenta la edad. A partir de los 55 años disminuye al 76,1\%, alcanzando el mínimo en el grupo de edad de 65 a 74 años (49,1\%).

Para Parsons (2014), el concepto de movilidad para alcanzar un aprendizaje más fluido, adaptativo, colaborativo y exploratorio es fundamental, sin embargo, se imponen barreras en tres niveles:

1. Normativas educativas sobre la restricción del uso de los teléfonos móviles en el aula.

2. Falta de conciencia por parte del alumnado de la utilidad educativa del teléfono móvil y no meramente con fines lúdicos.

3. Desconocimiento de la potencialidad educativa de las aplicaciones móviles por parte de los docentes, considerándolos en ocasiones elementos disruptivos y de intromisión en el aula.

Normativas, falta de conciencia del alumnado y desconocimiento de los docentes impiden, entre otros factores, que un medio universal en la actualidad como el teléfono móvil acceda al aula como potencial herramienta de enseñanza y aprendizaje. La Unesco (2013) planteó la elaboración de unas directrices para las políticas de aprendizaje móvil, en las que se pide, entre otras cosas, capacitar a los docentes para que impulsen el aprendizaje mediante tecnologías móviles. Entre las mencionadas directrices, encontramos las siguientes:

1. Dar prioridad al desarrollo profesional de los docentes. El éxito del aprendizaje móvil depende de la capacidad de los profesores para sacar el máximo partido de las ventajas pedagógicas que ofrecen los dispositivos móviles.

2. Proporcionar la capacitación técnica y pedagógica necesaria a los docentes al introducir soluciones y oportunidades de aprendizaje móvil. A medida que los dispositivos van evolucionando, se vuelven más difíciles de utilizar.

3. Alentar a los centros de capacitación de docentes a que incorporen el aprendizaje móvil en sus programas y planes de estudio.

4. Ofrecer a los educadores oportunidades para que compartan estrategias a fin de integrar eficazmente la tecnología en instituciones con recursos y necesidades similares. 

EL PENSAMIENTO DE LOS DOCENTES SOBRE EL USO FORMATIVO DEL TELÉFONO MÓVIL

En definitiva, para aprovechar al máximo las potencialidades de los teléfonos móviles inteligentes, es necesario que los docentes puedan incorporarlos con éxito en la práctica educativa, ya que «sin orientación e instrucciones, utilizarán la tecnología para enfocar las clases igual que siempre, pero añadiendo un soporte tecnológico, en lugar de transformar y mejorar los enfoques de la enseñanza y aprendizaje» (Vázquez-Cano y Sevillano, 2019).

No obstante, tampoco debemos olvidar que el uso de tales dispositivos en el contexto educativo, además de las fortalezas mencionadas, posee un conjunto de debilidades que hemos de tener presentes para tratar de aminorar. Mencionamos algunas:

- Elevado coste económico de la tarifa de acceso a datos para su conexión a internet.

- Para el uso de las apps específicas, se hace necesario poseer una buena cobertura para la conexión por datos a internet, o bien, tener acceso a alguna wifi con buen ancho de banda.

- Obsolescencia de los equipos al poco tiempo de uso.

- Limitaciones tecnológicas derivadas del manejo de equipos de reducido tamaño, con pantallas y teclados poco ergonómicos.

- Restricciones a su uso en los centros educativos.

- Inexistencia de un criterio consensuado de uso ante las miles de opciones que se ofrecen como software para dispositivos móviles.

Aunque venimos detallando varios de los motivos por los que los dispositivos móviles no han sido incorporados en las aulas como una herramienta más de enseñanza y aprendizaje, existen ejemplos que muestran inquietud y experimentación haciendo uso de dichas herramientas en diversos contextos educativos. Tenemos a nuestro alcance varias recopilaciones de experiencias de aprendizaje a través de dispositivos móviles o m-learning (Vázquez-Cano y Sevillano, 2019), destacan, por ejemplo, en España las basadas en proyectos piloto como PICAA. En otros muchos países del mundo también se han puesto en marcha este tipo de iniciativas. Podemos encontrar estudios sobre el uso educativo de los dispositivos móviles, con una amplia difusión internacional, pero aún no son suficientes las investigaciones dedicadas específicamente al teléfono móvil a pesar de la ya mencionada masiva presencia social en el entorno extraescolar.

Las limitaciones de uso en los centros educativos obliga a que se apoyen en la ruptura con los espacios educativos, siendo mayormente utilizados en las salidas escolares y en muchos casos las experiencias educativas están más ligadas a la educación no formal que a la formal. Los propios fabricantes y creadores de apps para móviles reconocen las múltiples posibilidades que ofrecen estos dispositivos para la enseñanza y aprendizaje, es más, muestran su creciente interés en que, como docentes, podamos desarrollar todo su potencial en el sector educativo. Estudian la forma de crear aplicaciones muy amigables, que presentan una excelente experiencia 
de uso, buscando que la curva de aprendizaje necesaria para para poder aplicar su uso en las aulas sea lo menos pronunciada posible. Por todo ello, nos encontramos con eventos educativos en los que están unificados los fabricantes de móviles con profesionales de la educación, como, por ejemplo, el denominado \#HuaweiEducación, sus participantes muestran las mismas inquietudes que venimos exponiendo en este documento, con intervenciones como, por ejemplo, la de Pablo Wang (2019, @Pablo_Wang_HW, director de la Unidad de Negocio de Huawei Consumo en España), que, entre otras cosas, afirma lo siguiente: «La generación que está ahora mismo en los colegios es nativa digital: no necesita explicaciones técnicas sobre cómo encender los dispositivos, sino que le enseñemos cómo usarlos con responsabilidad y así explotar su potencial». Durante nuestro trabajo de recopilación de fuentes, localizamos el artículo de la revista Didáctica, Innovación y Multimedia (DIM): «Las potencialidades del teléfono móvil como recurso innovador en el aula: una revisión teórica» (Gómez Hernández y Monge López, 2013), que nos ha guiado en nuestra labor de investigación sobre el estado de la cuestión y a sentar las bases de nuestro trabajo de investigación. Además, localizamos una investigación muy similar a la que queremos plantearnos realizada por Brazuelo Grund et al. (2016), por lo tanto, con nuestro trabajo, realmente trataremos de complementar y continuar la tarea ya emprendida por estos docentes, publicada en un artículo de la revista RED: Revista de Educación a Distancia titulado "Los docentes ante la integración educativa del teléfono móvil en el aula». En conclusión, desde el ámbito de la educación formal y no formal, se pueden usar los teléfonos móviles inteligentes como recurso extendido y atractivo entre los estudiantes, que pueden aprovechar el acceso que ofrecen desde cualquier lugar y en cualquier momento a internet para realizar actividades de formación. Teniendo en cuenta, además, que la investigación educativa se realiza para dar solución a un problema que tenemos o para saber más sobre algo que desconocemos del entorno educativo (López Fuentes y Salmerón Vílchez), la presente investigación va dirigida al cuerpo de docentes de Enseñanza Secundaria Obligatoria (ESO) y Formación Profesional (FP) y sus hábitos y costumbres utilizando el teléfono móvil, así como las perspectivas de un posible uso educativo del mismo. Los docentes deben ser los auténticos líderes educativos en la integración de las Tic en el aula (Brazuelo Grund et al., 2016).

\section{DISEÑO DE LA INVESTIGACIÓN}

La presente investigación se dirigió al cuerpo de docentes de Enseñanza Secundaria Obligatoria (ESO) y Formación Profesional (FP) de Castilla y León y sus hábitos y costumbres utilizando el teléfono móvil, así como las perspectivas de un posible uso educativo del mismo. El estudio realizado es de tipo exploratorio, descriptivo y correlacional. 
La perspectiva metodológica utilizada se enmarca dentro del paradigma interpretativo que persigue la comprensión de los fenómenos educativos a través del análisis de las percepciones e interpretaciones de los sujetos que intervienen en la acción educativa (García Llamas et al., 2001). Con este diagnóstico se pretende llevar a cabo el hallazgo de información que sirva como punto de partida para futuras investigaciones de mayor concreción y propuestas de mejora.

\subsection{Objetivos}

La finalidad general de la investigación consiste en analizar el uso que el docente de ESO y FP hace del móvil y la visión que tiene respecto a su incorporación como herramienta de trabajo en el aula. Esta cuestión genera mucha controversia, ya que hay reglamentos en centros que lo prohíben. Para dar respuesta al problema de investigación, nos propusimos los siguientes objetivos específicos:

1. Identificar la recepción de los docentes de ESO, Bachillerato y FP respecto al uso educativo del teléfono móvil en el aula.

2. Analizar el uso del teléfono móvil y sus aplicaciones por parte de los docentes de ESO, Bachillerato y FP.

3. Determinar posibles usos del teléfono móvil en el aula teniendo presentes tanto las ventajas como los inconvenientes de su incorporación.

\subsection{Muestra}

La muestra la constituyeron 42 profesores.

- La media de edad se encuentra entre los 38 y 54 años (59,5\%).

- Las mujeres superan a los hombres (71,4\% y 28,6\%).

- En cuanto a la antigüedad docente, el 78,6\% tiene al menos 10 años de experiencia e incluso el 47,6\% más de 20 años.

- El lugar del centro de trabajo que predomina es la provincia de Burgos, seguida de Valladolid, Palencia, Segovia, Zamora y Madrid.

- Por materias, se distribuyen en Lengua extranjera (17,9\%), Matemáticas y Lengua Española (15,4\% en ambos casos), Ciencias Sociales $(12,8 \%)$, Ciencias Naturales (10,4\%), Informática y Comunicaciones $(7,7 \%)$ y Tecnología $(5,1 \%)$. El resto de respuestas se corresponde a materias como Educación Física, Música, entre otras.

\subsection{Instrumentos de recogida de información}

El cuestionario lo elaboramos de forma digital para poder aplicarlo de forma virtual y con el fin de intentar captar más respuestas. El instrumento original consta de 90 ítems estructurados en 10 dimensiones incluyendo los aspectos más relevantes derivados del análisis de las fuentes documentales (estudios y experiencias). 
También se incluye un apartado de respuesta abierta para indicar las posibilidades educativas del teléfono móvil para cada una de las funcionalidades incluidas en la dimensión Valoración del teléfono móvil como herramientaeducativa.

\section{TABLA 1}

Dimensiones del cuestionario, n. ${ }^{\circ}$ de ítems, Alpha de Cronbach

\begin{tabular}{lccc}
\hline Dimensiones & N. ${ }^{\circ}$ ítemss & $\begin{array}{c}\text { Alpha de } \\
\text { Cronbach }\end{array}$ & Magnitud \\
\hline $\begin{array}{l}\text { Hábitos de consumo, posibilidades } \\
\text { multimedia, ofimáticas y de conexión }\end{array}$ & 26 & 0.7622 & Alta \\
\hline $\begin{array}{l}\text { Nivel general de conocimientos previos en } \\
\text { TIC }\end{array}$ & 3 & 0.948 & Muy alta \\
\hline $\begin{array}{l}\text { Nivel general de conocimientos técnicos } \\
\text { sobre telefonía móvil }\end{array}$ & 13 & 0.822 & Muy alta \\
\hline $\begin{array}{l}\text { Ambientes y usos del teléfono móvil en el } \\
\text { centro educativo y en el aula }\end{array}$ & 7 & 0.610 & Alta \\
\hline $\begin{array}{l}\text { Políticas educativas de prohibición de los } \\
\text { teléfonos móviles }\end{array}$ & 5 & 0.741 & Alta \\
\hline $\begin{array}{l}\text { Motivos y actitudes a favor del uso educativo } \\
\text { del teléfono móvil }\end{array}$ & 13 & 0.783 & Alta \\
\hline $\begin{array}{l}\text { Obstáculos y actitudes en contra de la } \\
\text { incorporación educativa del teléfono móvil }\end{array}$ & 7 & 0.693 & Alta \\
\hline $\begin{array}{l}\text { Valoración del teléfono móvil como } \\
\text { herramienta educativa }\end{array}$ & 18 & 0.896 & Muy alta \\
\hline
\end{tabular}

Adaptado de Brazuelo Grund, Gallego Gil y Cacheiro González (2016).

\section{ANÁLISIS DE RESULTADOS Y DISCUSIÓN}

Para comenzar el análisis de resultados, hemos cerrado la posibilidad de enviar más respuestas a nuestro formulario online, el cual hemos elaborado con la herramienta Formularios de Google. Esta herramienta nos da la posibilidad de consultar los resultados de uno en uno y también nos ofrece un resumen, compuesto, principalmente, por diferentes tipos de gráficos. Una vez que hemos importado los datos, revisamos las propiedades de las variables y modificamos las que consideramos necesarias. Tras la importación de datos, el programa spss nos ha clasificado todas las variables como nominales o categóricas salvo las correspondientes a edad, número de móviles, etc., que las ha clasificado de escala al ser numéricas.

En nuestro trabajo de revisión de propiedades de las variables, hemos convertido muchas de las nominales en variables ordinales, añadiendo las etiquetas de valor correspondientes al cuestionario creado inicialmente. También hemos etiquetado 
todas las variables posibles, para que, de este modo, la salida de datos tras el análisis tenga un resultado más fácil de interpretar. A las variables etiquetadas, además, se les ha dado un tipo de datos numérico para crear las categorías de modo más preciso.

Una vez revisadas y modificadas las propiedades de las variables y depurados los datos, procedemos al análisis de los resultados. Para llevar a cabo dicho análisis, trabajamos con métodos cuantitativos, que son los que mejor se ajustan a los resultados de cuestionarios. Los métodos cuantitativos aplicados son de diversa índole, teniendo en cuenta que utilizando tanto métodos univariados como bivariados obtendremos la mayor información posible.

\section{ANÁlISIS DE DATOS UNIVARIADO}

Comenzamos realizando el análisis univariado. Extraemos conclusiones a través de las frecuencias de respuesta y los porcentajes asociados a las mismas.

\subsection{Hábitos de consumo, posibilidades multimedia, ofimáticas y de conexión}

- Del total de los 42 encuestados solamente dos de ellos afirman que llevan teniendo teléfono móvil desde hace 4 o 6 años, los 40 restantes lo tienen desde hace más de 6 años.

- La media de móviles que han tenido hasta ahora son 5,36, teniendo esta variable un valor mínimo de 2 (persona que menos móviles ha tenido hasta ahora) y un máximo de 10 (persona que más móviles ha tenido hasta ahora) con una mediana de 5.

- La totalidad de los encuestados afirman tener en el móvil las siguientes aplicaciones: cámara de vídeo, reproductor de audio, de vídeo, internet y GPS.

- Solamente uno de los encuestados afirma no saber si su teléfono móvil tiene alguna de las siguientes aplicaciones, el resto han respondido que sí disponen de ellas: cámara de fotos, grabadora de sonidos, wifi y modo avión.

- Dos de los individuos afirman que no saben si su móvil puede abrir archivos Word o tiene Bluetooth. Los 40 restantes afirman disponer de dichas aplicaciones

- Para el resto de características nos encontramos con mucha variedad de respuestas.

La frecuencia con la que realizan cursos de formación en Tic está repartida: cada año el 30,95\%, cada dos años otro 30,95\%, cada tres años o más el 35,71\% y un $2,38 \%$ no lo realizan nunca: 


\section{TABLA 2}

Nivel general de conocimientos técnicos sobre telefonía móvil

\begin{tabular}{|c|c|c|c|c|}
\hline & $\begin{array}{l}\text { No sé lo que } \\
\text { significa }\end{array}$ & $\begin{array}{l}\text { Sé lo que } \\
\text { es pero no } \\
\text { sé hacerlo }\end{array}$ & $\begin{array}{c}\text { Puedo hacerlo } \\
\text { con ayuda de } \\
\text { alguien }\end{array}$ & $\begin{array}{l}\text { Puedo } \\
\text { hacerlo sin } \\
\text { problemas }\end{array}$ \\
\hline Hacer una foto & & & $4,8 \%$ & $88,1 \%$ \\
\hline Grabar un vídeo & & & $4,8 \%$ & $85,7 \%$ \\
\hline $\begin{array}{l}\text { Enviar un mensaje de } \\
\text { texto }\end{array}$ & & & $4,8 \%$ & $88,1 \%$ \\
\hline $\begin{array}{l}\text { Utilizar un buscador de } \\
\text { internet }\end{array}$ & & & $4,8 \%$ & $88,1 \%$ \\
\hline $\begin{array}{l}\text { Crear un PowerPoint } \\
\text { para móviles }\end{array}$ & & $19,0 \%$ & $26,2 \%$ & $47,6 \%$ \\
\hline $\begin{array}{l}\text { Diseñar una página web } \\
\text { para móviles }\end{array}$ & $4,8 \%$ & $45,2 \%$ & $21,4 \%$ & $21,4 \%$ \\
\hline Crear un podcast & $9,5 \%$ & $45,2 \%$ & $14,3 \%$ & $21,4 \%$ \\
\hline $\begin{array}{l}\text { Enviar un email a través } \\
\text { del teléfono móvil }\end{array}$ & & & $7,1 \%$ & $85,7 \%$ \\
\hline $\begin{array}{l}\text { Guardar archivos en el } \\
\text { teléfono móvil }\end{array}$ & $2,4 \%$ & $4,8 \%$ & $7,1 \%$ & $78,6 \%$ \\
\hline Escuchar música & & $2,4 \%$ & $9,5 \%$ & $81,0 \%$ \\
\hline Utilizar el Bluetooth & $2,4 \%$ & & $7,1 \%$ & $78,6 \%$ \\
\hline $\begin{array}{l}\text { Compartir archivos por } \\
\text { el teléfono móvil }\end{array}$ & $2,4 \%$ & $4,8 \%$ & $7,1 \%$ & $78,6 \%$ \\
\hline
\end{tabular}

Como se puede apreciar las tareas de uso más cotidiano con el teléfono móvil, como hacer una foto, enviar mensajes, etc., tienen respuestas positivas de forma masiva.

\subsection{Ambientes y usos del teléfono móvil en el centro educativo y en el aula}

El 88,1\% de los encuestados afirman llevar el móvil siempre al centro educativo y un $9,5 \%$ dicen llevarlo casi siempre, aunque los porcentajes cambian si hablamos de su utilización en el centro educativo: el 47,6\% lo utiliza a veces, el 19\% siempre, el 11, 9\% casi siempre, el 7,1\% casi nunca y otro $11,9 \%$ no lo utiliza nunca. De estos datos podemos concluir que todos los profesores lo llevan al centro educativo, pero algunos apenas lo utilizan.

Los lugares elegidos mayoritariamente para su uso son la sala de profesores y el recreo.

El 83,3\% afirma conocer alguna experiencia de uso educativo del teléfono móvil y el 16,7\% restante no conoce ninguna. De los que sí que las conocen y nos responden a la breve explicación de la misma, muchos mencionan la aplicación Kahoot, famosa porque permite gamificar el aula creando cuestionarios de evaluación y 
responderlos como si de un concurso se tratara. Otra de las experiencias que más repiten es para la lectura de códigos QR y la búsqueda de información. A la pregunta ¿Te gustaría conocer y aplicar el teléfono móvil con fines didácticos en el aula?, el $66,67 \%$ muestra interés en conocer este tipo de experiencias.

\subsection{Politicas educativas de prohibición de los teléfonos móviles}

En cuanto a la dimensión del cuestionario relacionada con las políticas educativas de prohibición de los teléfonos móviles, vemos el siguiente diagrama.

\section{FIGURA 3}

Prohibición del uso del móvil en los centros educativos

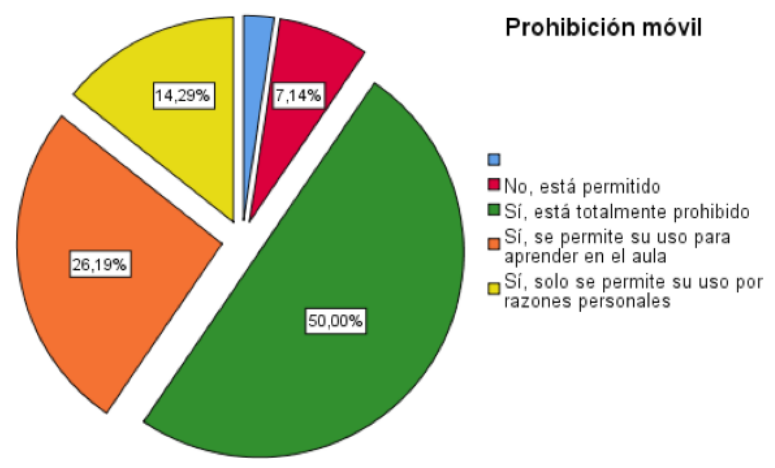

También incluimos otro diagrama de sectores que muestra los resultados obtenidos cuando se les pregunta si creen que la norma sobre la prohibición de los teléfonos móviles se cumple.

\section{FIGURA 4}

Cumplimiento de la norma de la prohibición de los móviles

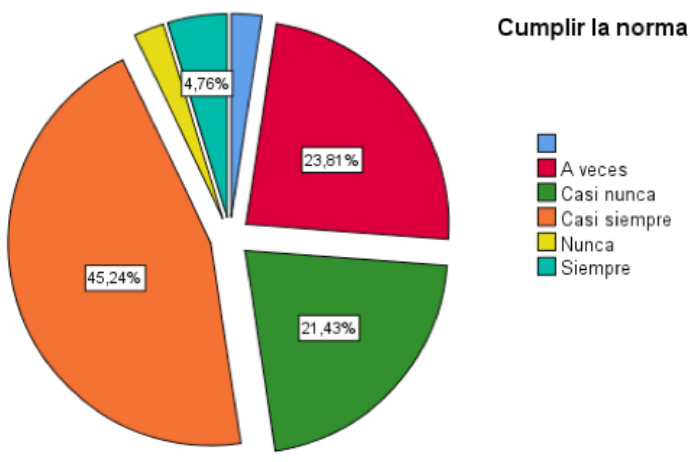




\subsection{Motivos y actitudes a favor del uso educativo del teléfono móvil}

Los datos disponibles sobre la dimensión motivos y actitudes a favor del uso educativo del teléfono móvil quedan resumidos en la siguiente tabla:

\section{TABLA 3}

Motivos y actitudes a favor del uso educativo del teléfono móvil

\begin{tabular}{lccc}
\hline & $\begin{array}{c}\text { Totalmente } \\
\text { de acuerdo y } \\
\text { de acuerdo }\end{array}$ & $\begin{array}{c}\text { Ni de } \\
\text { acuerdo ni en } \\
\text { desacuerdo }\end{array}$ & $\begin{array}{c}\text { En desacuerdo } \\
\text { y totalmente en } \\
\text { desacuerdo }\end{array}$ \\
\hline $\begin{array}{l}\text { Dispositivo al alcance de todos } \\
\text { alumnos }\end{array}$ & $\mathbf{7 6 , 2 \%}$ & $9,5 \%$ & $14,3 \%$ \\
\hline Grandes capacidades multimedia & $\mathbf{8 8 , 1 \%}$ & $9,5 \%$ & \\
\hline Consultar internet & $\mathbf{9 2 , 9 \%}$ & $2,4 \%$ & $4,8 \%$ \\
\hline $\begin{array}{l}\text { Alumnado necesita formación para } \\
\text { su utilización }\end{array}$ & $47,6 \%$ & $7,1 \%$ & $\mathbf{4 2 , 8 \%}$ \\
\hline $\begin{array}{l}\text { Aprendizaje en todo momento y } \\
\text { lugar }\end{array}$ & $57,2 \%$ & $21,4 \%$ & $19,1 \%$ \\
\hline Facilita comunicación con familias & $69,0 \%$ & $17,6 \%$ & $11,9 \%$ \\
\hline Favorece feedback alumno profesor & $\mathbf{4 0 , 5 \%}$ & $33,3 \%$ & $23,8 \%$ \\
\hline Favorece aprendizaje colaborativo & $54,8 \%$ & $31,0 \%$ & $9,5 \%$ \\
\hline $\begin{array}{l}\text { Puede abrir nuevas oportunidades } \\
\text { aprendizaje }\end{array}$ & $\mathbf{7 6 , 2 \%}$ & $11,9 \%$ & $9,5 \%$ \\
\hline Más económico que ordenador & $47,7 \%$ & $23,8 \%$ & $26,2 \%$ \\
\hline Motivante para el alumnado & $69,0 \%$ & $19,0 \%$ & $9,6 \%$ \\
\hline Útil para gestión del centro & $52,4 \%$ & $23,8 \%$ & $21,5 \%$ \\
\hline $\begin{array}{l}\text { A favor de uso educativo móvil solo } \\
\text { fuera del aula }\end{array}$ & $35,7 \%$ & $19,0 \%$ & $\mathbf{4 2 , 9 \%}$ \\
\hline
\end{tabular}

La tendencia general de los resultados muestra una actitud positiva en todos los ítems hacia la incorporación en el aula del teléfono móvil como recurso educativo. Destacan positivamente los ítems Podemos consultar información en internet a través del móvil con un 92,9\% totalmente de acuerdo y de acuerdo y Posee grandes capacidades multimedia con un 88,1\%. También destacan positivamente los ítems El teléfono móvil es un dispositivo al alcance de todo el alumnado y Puede abrir nuevas oportunidades de aprendizaje, ambos con un 76,2\%. Sin embargo, en el ítem Favorece el feedback entre alumno y profesor, tenemos el porcentaje más bajo de todos en la opción totalmente de acuerdo y de acuerdo con un 40,5\%. 


\section{TABLA 4}

Obstáculos y actitudes en contra de la incorporación educativa del teléfono móvil

\begin{tabular}{lccc}
\hline & $\begin{array}{c}\text { Totalmente de } \\
\text { acuerdo y de } \\
\text { acuerdo }\end{array}$ & $\begin{array}{c}\text { Ni de acuerdo ni } \\
\text { en desacuerdo }\end{array}$ & $\begin{array}{c}\text { En desacuerdo } \\
\text { y totalmente en } \\
\text { desacuerdo }\end{array}$ \\
\hline $\begin{array}{l}\text { Interrumpe clases con } \\
\text { llamadas o mensajes }\end{array}$ & $\mathbf{7 3 , 8 \%}$ & $11,9 \%$ & $11,9 \%$ \\
\hline $\begin{array}{l}\text { Pantalla pequeña } \\
\text { Temor a fotos o } \\
\text { grabaciones no } \\
\text { consentidas }\end{array}$ & $\mathbf{6 1 , 9 \%}$ & $28,6 \%$ & $7,2 \%$ \\
\hline $\begin{array}{l}\text { El móvil es un juguete, } \\
\text { no una herramienta de } \\
\text { aprendizaje }\end{array}$ & $\mathbf{6 9 , 1 \%}$ & $16,7 \%$ & $11,9 \%$ \\
\hline $\begin{array}{l}\text { Ratio alumnado no } \\
\text { permite su integración en } \\
\text { el aula }\end{array}$ & $23,8 \%$ & $28,6 \%$ & $\mathbf{4 5 , 2 \%}$ \\
\hline $\begin{array}{l}\text { Problemas de cobertura } \\
\text { Costoso económicamente }\end{array}$ & $45,2 \%$ & $31,0 \%$ & $21,4 \%$ \\
\hline para el alumnado & $19,1 \%$ & $23,8 \%$ & $33,3 \%$ \\
\hline
\end{tabular}

A raíz de los resultados obtenidos, podemos comprobar cuáles son las dudas o temores más comunes entre los docentes a la hora de incorporar el teléfono móvil como herramienta educativa en el aula. Con un 73,8\%, el ítem interrumpe las clases con llamadas o recepción de mensajes representa el valor más alto en la opción totalmente de acuerdo y de acuerdo, le siguen Temo que me hagan fotos o me graben con el teléfono móvil sin mi consentimiento con un 69,1\% y la pantalla de un teléfono móvil es demasiado pequeña con un $61,9 \%$.

\subsection{Valoración del teléfono móvil como berramienta educativa}

Para la última dimensión del cuestionario en la cual se pide valorar las diferentes funcionalidades que ofrece el móvil pensando en su enfoque educativo, obtenemos los siguientes resultados.

Podemos comprobar que las funcionalidades que se consideran de mayor utilidad son internet $(90,5 \%)$, email (85,7\%), apps específicas $(83,4 \%)$, notas $(83,3 \%)$ y calculadora (81,0\%). Las consideradas de menor utilidad, pero con mucho menos apoyo que las anteriores, son llamadas $(28,5 \%)$, radio $(16,7 \%)$, reproductor de música y mensajes instantáneos (ambos ítems con un 14,3\%). 


\section{TABla 5}

Valoración del teléfono móvil como herramienta educativa

\begin{tabular}{|c|c|c|c|}
\hline & Muy útil y útil & Indiferente & Poco útil y nada útil \\
\hline Llamadas & $42,9 \%$ & $28,6 \%$ & $28,5 \%$ \\
\hline Mensajes instantáneos & $61,9 \%$ & $23,8 \%$ & $14,3 \%$ \\
\hline Internet & $90,5 \%$ & $4,8 \%$ & $4,8 \%$ \\
\hline GPS & $61,9 \%$ & $26,2 \%$ & $11,9 \%$ \\
\hline Email & $85,7 \%$ & $11,9 \%$ & $2,4 \%$ \\
\hline Calendario & $78,6 \%$ & $19,0 \%$ & $2,4 \%$ \\
\hline Reloj & $59,5 \%$ & $33,3 \%$ & $7,1 \%$ \\
\hline Cronómetro & $59,5 \%$ & $31,0 \%$ & $7,2 \%$ \\
\hline Calculadora & $81,0 \%$ & $14,3 \%$ & $2,4 \%$ \\
\hline Notas & $83,3 \%$ & $9,5 \%$ & $4,8 \%$ \\
\hline Grabadora sonidos & $65,3 \%$ & $23,8 \%$ & $11,9 \%$ \\
\hline Reproductor música & $57,1 \%$ & $28,6 \%$ & $14,3 \%$ \\
\hline Radio & $52,4 \%$ & $31,0 \%$ & $16,7 \%$ \\
\hline Cámara fotos & $76,2 \%$ & $14,3 \%$ & $7,2 \%$ \\
\hline Cámara vídeo & $71,4 \%$ & $21, .4 \%$ & $7,2 \%$ \\
\hline USB & $81,0 \%$ & $11,9 \%$ & $7,2 \%$ \\
\hline Tarjeta memoria & $73,8 \%$ & $14,3 \%$ & $4,8 \%$ \\
\hline Apps específicas & $83,4 \%$ & $9,5 \%$ & $2,4 \%$ \\
\hline
\end{tabular}

\subsection{Posibilidades educativas del teléfono móvil desde las disciplinas impartidas}

Para terminar con el análisis descriptivo univariado, presentamos las respuestas más relevantes que hemos obtenido a través de la pregunta abierta del cuestionario sobre las posibilidades educativas del teléfono móvil desde la materia que imparte cada profesor. Señalaremos las siguientes, teniendo en cuenta que muchas de las respuestas se encontraban de forma repetida o muy similar:

\section{- Llamadas}

- Comunicación con compañeros que formamos parte de un proyecto educativo común. 
- Llamas en roleplay.

- De auxilio 112 o 091.

- Contacto con familias.

- Mensajes instantáneos

- Para avisos, en extraescolares, cambios imprevistos, compartir enlaces...

- Feedback.

- Recordatorios o dudas.

- Prácticas de escritura.

- Información, contenidos y actividades de refuerzo y/o ampliación, actividades enigma.

- Para dar soluciones o respuestas a ejercicios, para brainstorming.

- $\quad G P S$

- Cuando realizamos salidas del centro.

- Geografía, Geología, Matemáticas.

- Unidad de planos y escalas.

- En las unidades didácticas de orientación.

- Cálculo de coordenadas.

- Actividades que incluyan la geolocalización, utilidades a partir de los sistemas de información geográfica.

- Recorridos didácticos.

- Para aprender a orientarse.

- Estudiamos orientación, mapas geológicos, mapas de biodiversidad...

- Email

- Para enviar o recibir trabajos.

- Feedback y entrega de trabajos.

- Comunicación con padres y alumnos.

- Trabajo cooperativo.

- Comunicaciones semioficiales, apoyo educativo al alumnado.

- Transmisión de archivos y noticias.

- Avisos e información útil.

- Comunicación con el alumnado, envío de material...

\section{- Calendario}

- Para planificar y programar el curso escolar, proyectos, etc.

- Fechas de trabajos, exámenes...

- Fechas importantes. Sustituir la agenda por este.

- Organización del alumno.

- Organización y planificación de trabajos y proyectos.

- Planificación de eventos escolares.

- Reloj

- Para medir los tiempos de las tareas.

- Para controlar la hora. 
- Cronómetro

- Alguna actividad cronometrada.

- Para speaking o controlar tiempos.

- Para la toma de tiempos en educación física en momentos determinados.

- Prácticas de laboratorio.

- Control de tiempo de actividades.

- Para concursos, exposiciones orales.

- Calculadora

- Para sustituir la calculadora tradicional.

- Notas y operaciones.

- Resolver problemas.

- Operaciones más complicadas.

- Matemáticas.

- Exámenes.

- Realizar operaciones de manera rápida.

- Cálculo de notas.

- Notas

- Para ir tomando nota de tareas pendientes.

- Cualquier nota importante.

- Para guardar anotaciones.

- Deadline, apuntes concretos.

- Apuntes de anécdotas, citas, apuntar actividades.

- Apuntes y material importante.

- Funciona como agenda.

- Para apuntar ejemplos, excepciones, tareas...

- Apuntar avisos importantes.

- Grabadora de sonidos

- Grabar entrevistas.

- Speaking.

- Grabación de ejercicios de fonética, de diálogos entre alumnos.

- Presentaciones.

- Roleplay.

- Notas de audio actitudinales o alguna cosa concreta acontecida en clase.

- Grabar exposiciones orales.

- Actividades tipo resumen para mejorar la expresión oral.

- Salidas fuera del centro.

- En trabajos o reuniones que se considere necesario.

- Para grabarse o grabar otros alumnos en presentaciones para una corrección detenida.

- En salidas al campo. 
- Reproductor de música

- Reproducir podcast, entrevistas grabadas...

- Listening.

- Ambiente, días especiales.

- Muy útil en el caso de educación física para calentamientos, vueltas a la calma y coreografías.

- Reforzar ciertas actividades.

- Muy interesantes en la materia de música y como música de ambiente cuando sea necesario.

- Para oír canciones en inglés o entrevistas, noticias o podcasts de los periódicos y radio de habla inglesa.

- En sesiones mindfulness.

- Radio

- Escuchar algún programa educativo.

- Listening, escucha de emisoras en lengua inglesa.

- Para realizar actividades que fomenten la escucha activa.

- Podcast, no radio en directo.

- Cámara de fotos

- Hacer fotos de las fases de proyectos educativos.

- Actividades relacionadas.

- Fotografiar animales, plantas, preparaciones microscópicas, paisajes, agentes geológicos...

- Restrictivo para descripción de fotos.

- Almacenar ideas importantes.

- En excursiones, eventos y días especiales.

- Presentaciones.

- Fotos para incluir en trabajos, para resaltar el entorno educativo, poner en valor alguna actividad realizada en clase.

- Salidas fuera del centro y actividades de laboratorio.

- Guardar imágenes significativas de cualquier proceso educativo, si se considera necesario.

- Comparativa de evolución de un ecosistema, identificación material biológico y geológico...

- Cámara de vídeo

- Grabar entrevistas, videotutoriales...

- Realizar vídeos explicativos, por ejemplo, de prácticas de laboratorio.

- Grabaciones speaking.

- Roleplay.

- Evaluación exposiciones orales.

- Grabar contenidos, exposiciones orales, vídeos para metodología flipped classroom. 
- Guardar información de procesos de aprendizaje o vivencias.

- Grabar diálogos en situaciones, crear cortos con cierta temática.

- Representaciones teatralizadas de descubrimientos biológicos, prácticas...

- USB

- Transferir los archivos del móvil al ordenador.

- Guardar archivos que ocupan mucha memoria del teléfono.

- Almacenamiento de datos o cosas que se quieran exponer en ordenadores de diferentes aulas.

- Tarjeta de memoria

- Almacenar todos los archivos que generemos con el móvil.

- Para tener espacio.

- Guardar archivos, fotos, vídeos.

- Compartir archivos.

- Almacenaje de apuntes, materiales...

- Apps específicas

- AppInventor: para crear aplicaciones móviles.

- Apps para el aprendizaje de idiomas, Gloster, Presentaciones...

- Quizlet. Crear unidades de estudio para que los alumnos puedan acceder y aprender. En clase jugar con los móviles al juego colaborativo de preguntas y respuestas.

- Kahoot, plickers, sonómetro, altímetro, Wikiloc...

- Yofindo.

\section{ANÁLISIS DE DATOS BIVARIADO}

Las pruebas no paramétricas que aplicamos se realizan con el objetivo de analizar diferentes tipos de influencia, relación o asociación entre dos variables. Las variables socioacadémicas que se han tenido en cuenta son: edad, materia impartida, nivel de conocimientos TIC e intención de utilizar el teléfono móvil como recurso educativo en el aula.

\subsection{Relación entre el nivel TIC y la edad de los sujetos}

En este caso la variable independiente es edad, que es una variable de intervalo, y la variable dependiente es nivel Tic, que es una variable ordinal. En este caso, al tener involucrada en la relación una variable ordinal, hemos de calcular un coeficiente de correlación apto para este tipo de variables, como, por ejemplo, la r de Spearman o la Tau-b de Kendall. Nosotros utilizaremos, por ejemplo, la $\mathrm{r}$ de Spearman.

Para visualizar gráficamente exponemos el diagrama de dispersión, que ilustra muy bien el grado de variación conjunta entre las variables. Tal y como puede verse, la nube de puntos y la recta de regresión muestran claramente la práctica 
ausencia de correlación entre ambas variables, aunque podemos comprobar que la ligera relación que se aprecia es negativa, que se interpreta de la siguiente manera: a mayor edad, menor nivel Tic.

\section{FIGURA 5}

Diagrama de dispersión de la edad y el nivel TIC

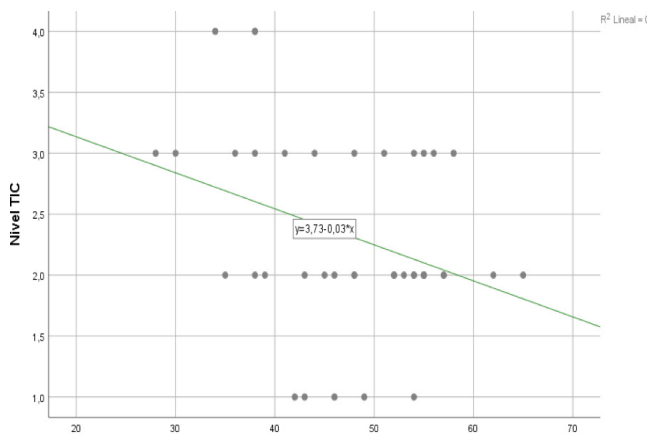

\subsection{Relación entre la edad y la intención de utilizar el móvil como recurso educativo en el aula}

En este caso tenemos la misma variable independiente y la variable dependiente es la intención de utilizar el móvil como recurso educativo en el aula, que es una variable ordinal. Es decir, estamos ante una situación muy similar a la anterior, por lo que haremos nuevamente la prueba $\mathrm{r}$ de Spearman. Correlación medio-baja, que nos viene a indicar que a mayor edad existe menor intención de integrar la utilización del teléfono móvil como recurso educativo en el aula.

\section{FIGURA 6}

Diagrama de dispersión de la edad y la intención del uso educativo del móvil

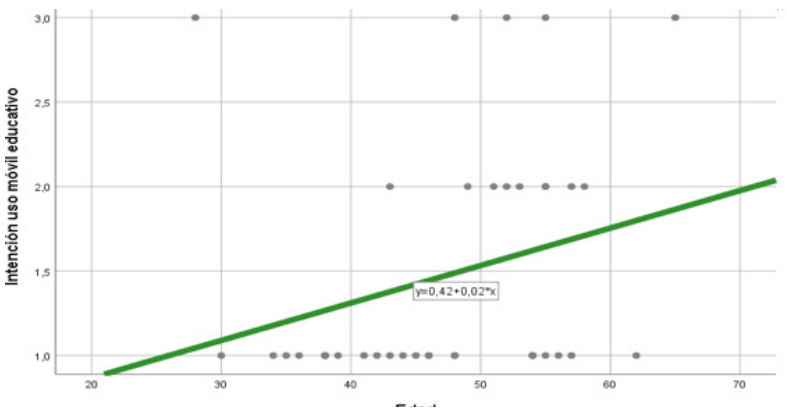




\subsection{Relación entre la materia impartida y el nivel TIC}

En este caso, la variable materia impartida es una variable nominal o categórica y la variable nivel TIC es una variable ordinal. Cuando buscamos la relación entre dos variables y una de ellas es nominal o categórica, propiamente lo que se calcula es la asociación entre ellas (no podemos hablar de correlación estrictamente). Por lo tanto, para estudiar la posible asociación ente nuestras variables, utilizaremos el procedimiento de las tablas cruzadas, que incluye el cálculo del estadístico de asociación Chi-Cuadrado y del Coeficiente de Contingencia (Gil Pascual, 2015), ya que este tipo de pruebas son las que se recomiendan para el tipo de variables con el que vamos a trabajar.

El Coeficiente de Contingencia entre ambas variables es $\mathrm{C}=0,692$; lo que indica una asociación alta, lo que ocurre es que no resulta ser estadísticamente significativa $(p=0,351>0,05)$. La prueba Chi-cuadrado, en este caso, contrasta la hipótesis nula $\mathrm{HO}=$ no hay asociación entre las variables (las variables son independientes entre sí). Como $\mathrm{p}=0,351>0,05 \rightarrow$ no se rechaza (se acepta) la hipótesis nula, por lo que no podemos afirmar que la asociación alta que nos ofrece el Coeficiente de Contingencia sea estadísticamente significativa entre ambas variables. Podemos ver la representación gráfica de la asociación estudiada en el siguiente gráfico de barras.

\section{FIGURA 7}

Relación entre el nivel TIC y la materia impartida

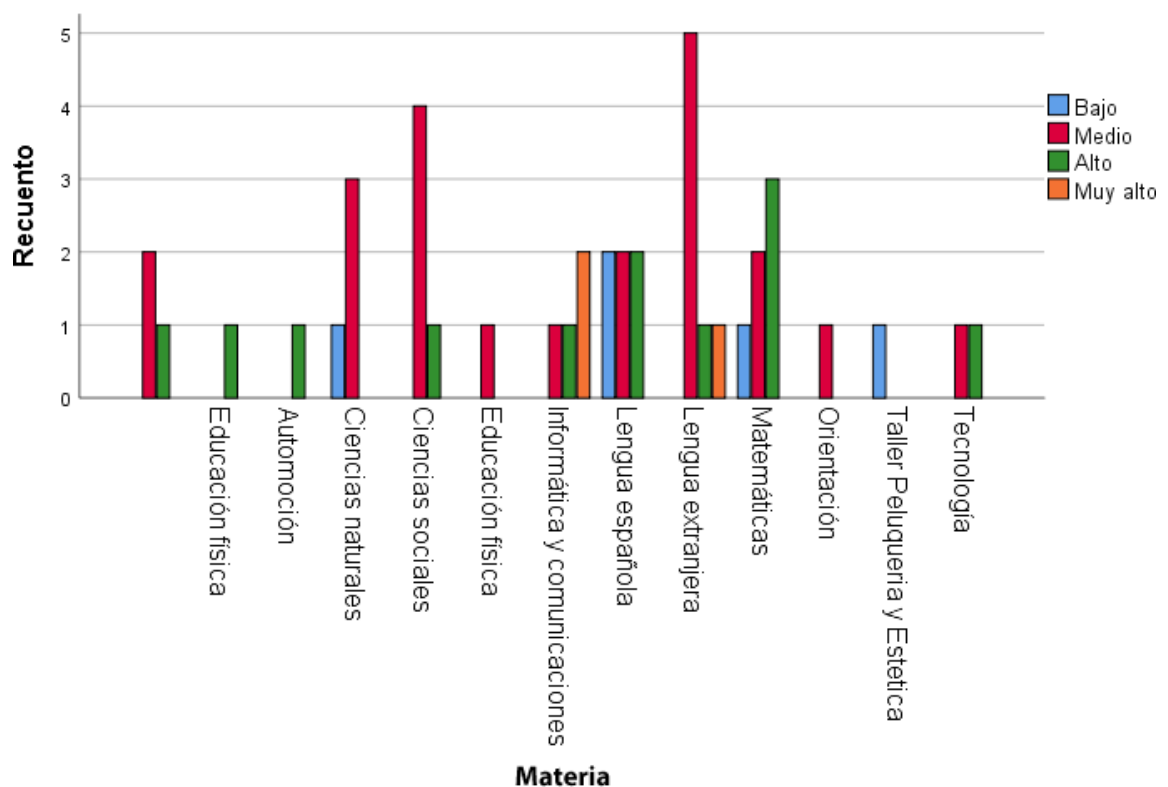




\subsection{Relación entre la materia impartida y la intención de utilizar el móvil como recurso educativo en el aula}

En este caso queremos estudiar la asociación entre la variable materia impartida, que es categórica, y la variable intención de utilizar el móvil en el aula, que es de tipo ordinal. El procedimiento a utilizar para estudiar esta asociación será de nuevo el de las tablas cruzadas, que incluye el cálculo del estadístico de asociación ChiCuadrado y del Coeficiente de Contingencia. El Coeficiente de Contingencia entre ambas variables es $\mathrm{C}=0,630$; lo que indica una asociación alta, como en el caso anterior, pero también ocurre que no resulta ser estadísticamente significativa ( $\mathrm{p}=$ $0,273>0,05)$, por lo que no podemos afirmar que la asociación alta que nos ofrece el Coeficiente de Contingencia sea estadísticamente significativa entre ambas variables.

Podemos ver la representación gráfica de la asociación estudiada en el siguiente gráfico.

\section{FIGURA 8}

Relación de la intención de uso educativo del móvil con la materia impartida

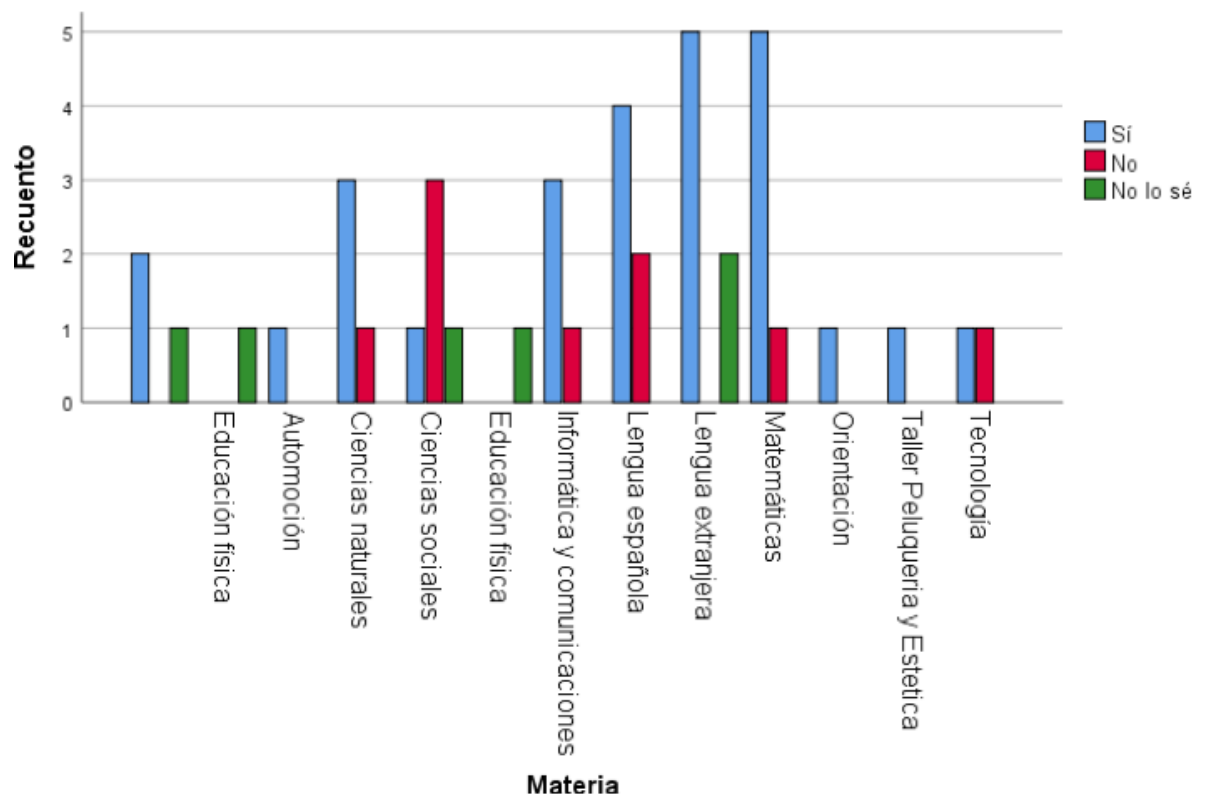




\section{Discusión}

Gracias a nuestro estudio, hemos podido crear una instantánea acerca de la situación del teléfono móvil en centros de secundaria y formación profesional. Hemos constatado que el teléfono móvil supone una potencial herramienta educativa, pero, a pesar de su impacto social y variadas prestaciones que nos ofrece, su uso pedagógico se halla limitado. La investigación muestra el valor que los docentes conceden hacia el teléfono móvil como recurso útil en contextos educativos. Lo consideran un dispositivo tecnológico que posee numerosas y variadas funcionalidades de conexión, ofimáticas y multimedia, que debería contar con un lugar entre las TIC aplicadas a educación. La actitud mostrada por los docentes es un factor favorecedor para la integración educativa del teléfono móvil. En este punto, vamos a resaltar las comparaciones más relevantes que hemos obtenido del resultado de nuestra investigación, respecto a los obtenidos por Brazuelo Grund et al. (2016). En nuestro estudio, el 83,3\% de los docentes afirman conocer alguna experiencia de uso educativo del teléfono móvil. Solamente el 16,7\% expresa desconocimiento de dichas prácticas educativas, frente al 93,3\% que mostraban Brazuelo Grund et al. (2016) a raíz de su investigación. En estos tres años las experiencias educativas que incorporan el uso del teléfono móvil están proliferando.

En estudios similares en intencionalidad y metodología (Sáez López, Sevillano García y Pascual Sevillano, 2019: 80) sobre aplicación del juego ubicuo con realidad aumentada en Educación Primaria, se concluye que una integración de estos diseños pedagógicos requiere de ciertos recursos, una estructura con buena conexión y una formación docente adecuada para integrarlo. Hallazgos que compartimos también en este estudio sobre el teléfono móvil. No obstante, no hemos de olvidar lo que señalaba Zapata-Ros (2015) ya que no se trata de evaluar el uso del dispositivo móvil como herramienta de aprendizaje, sino de las actividades en las que se ha incorporado para valorar su relevancia, pertinencia y ejecución en el transcurso de las mismas. Otro dato que nos gustaría destacar es el relativo a las políticas educativas de prohibición de los teléfonos móviles. En nuestra investigación hemos hallado que el 50\% se muestra de acuerdo con dichas políticas, frente al 78,5\% que mostraba Brazuelo Grund et al. (2016). Esta tendencia indica que los docentes valoran cada vez más el teléfono móvil como dispositivo de aprendizaje. Respecto a los motivos y actitudes para el uso educativo del teléfono móvil, los docentes lo perciben como un dispositivo al alcance de los alumnos, con grandes potencialidades multimedia y especialmente útil para la consulta de información a través de internet.

En esta dimensión nos encontramos dos ítems que, al comparar nuestros resultados con los de Brazuelo Grund et al. (2016), han variado especialmente. El primero es: puede abrir nuevas oportunidades de aprendizaje, cuyas respuestas positivas han ascendido de un 58,3\% al 76,2\%. El segundo sería que el alumnado no necesita formación para su utilización, en este caso es en las opciones en desacuerdo y totalmente en desacuerdo en las que se aprecia el cambio (del 28,3\% al 42,8\%).

En definitiva, los docentes ven cada vez más claramente que el dispositivo móvil ofrece potencialidades para su uso como herramienta de aprendizaje. Esta idea se 
constata aún más cuando observamos los resultados obtenidos para la dimensión obstáculos y actitudes en contra de la incorporación educativa del teléfono móvil, ya que el mayor cambio se produce en los resultados obtenidos por el ítem considero el teléfono móvil un juguete y no una berramienta de aprendizaje, el cual ha subido en la opción en desacuerdo y totalmente en desacuerdo del $28,7 \%$ obtenido en el estudio anterior al 45,2\% obtenido en el estudio actual.

Expondremos, a continuación, los resultados correspondientes a la valoración del teléfono móvil como herramienta educativa, que nos permite ver cuáles son las funcionalidades de los teléfonos móviles mejor valoradas. La totalidad de las funcionalidades han ascendido en popularidad para su uso educativo respecto de los datos recogidos por Brazuelo Grund et al. (2016), pero especialmente lo han hecho el uso de internet y el email. En nuestro estudio añadimos un ítem a esta dimensión, haciendo referencia al uso de apps móviles específicas para su uso educativo, que consideramos que han ayudado a una buena reputación para su uso educativo. Este hecho, además, se constata con las respuestas a la pregunta abierta del cuestionario, a través de la cual podían expresar si han utilizado alguna vez dichas funcionalidades. Pues bien, nos hemos encontrado que, en muchas de las experiencias descritas por los docentes, mencionan este tipo de apps específicas que estamos mencionando.

Tras realizar el análisis comparativo referente a los datos obtenidos con el análisis univariado, vamos a destacar lo referente al análisis bivariado. Los resultados reflejan que, con respecto a la edad, los docentes de menor edad consideran tener mayor nivel en conocimientos Tic que los de mayor edad, lo mismo ocurre con la predisposición a integrar la utilización del teléfono móvil como recurso educativo en el aula. Hemos detectado que los profesores de formación profesional de la familia de informática y comunicaciones, así como los de lengua extranjera, presentan niveles TIC más elevados, así como actitudes y motivos más a favor del uso de este dispositivo en el aula. Vemos una clara predisposición en los docentes a integrar el teléfono móvil como recurso educativo en el aula, lo consideran una herramienta de gran potencial que puede contribuir a la obtención de variadas competencias por parte de los alumnos. Esta tarea de integración supone también un cambio en las metodologías, cambios únicamente posibles a través de formación para los profesores, de este modo, los alumnos recibirán también la formación adecuada para su uso. Sin la colaboración de las familias, no llegaremos a conseguir este cambio de visión del teléfono móvil, ya que sería muy importante que desde las primeras veces que tienen este dispositivo conozcan las múltiples opciones que les ofrece y no lo utilicen como un mero objeto de ocio.

\section{Propuestas para la integración del teléfono MÓVIL EN El Aula}

A lo largo de este trabajo, hemos venido señalando el impacto social de la telefonía móvil entre la comunidad educativa (docentes, alumnado y familias) y la necesidad de un vínculo permanente entre sociedad y escuela, con respecto al uso 
de las tecnologías. Esta sinergia ha de reflejarse en cambios normativos. Prohibir un medio connatural y cotidiano como es el teléfono móvil es negar sus posibilidades educativas, dejando de lado una potencial herramienta educativa al servicio del aprendizaje

El principal motivo por el que los teléfonos móviles se han tratado de mantener fuera del contexto escolar es el hecho de ser consideradas como disruptivas o incitar a conductas inapropiadas. El desarrollo de actitudes del profesorado orientadas a fomentar la confianza y vencer las resistencias depende del conocimiento que se tenga de la tecnología en el ámbito educativo en el que se pretenda aplicar.

Una adecuada integración de estos dispositivos como herramienta de enseñanza y aprendizaje exige no solo avances técnicos, sino propuestas de actuación. Con nuestro estudio apoyamos las necesidades que, para hacer posible dicha integración, propusieron Brazuelo Grund et al. (2016) en su investigación y añadimos algunos detalles tres años después:

- Difusión del uso educativo de las tecnologías móviles o m-learning, mediante iniciativas que fomenten publicaciones que compartan experiencias y proyectos que puedan ser tomados como ejemplo por otros.

- Creación de contenidos específicos y realización de adaptaciones metodológicas aprovechando las posibilidades y características de los dispositivos móviles.

- Formación del profesorado que enseñe a los docentes cómo integrar los dispositivos móviles en su quehacer diario con un enfoque práctico e integrador para mejorar los procesos de enseñanza.

- Incorporación del teléfono móvil dentro de la lista de recursos TIC recomendados para utilizar en las aulas que imponen las normativas de los currículos de cada comunidad autónoma.

- Cambios en los reglamentos de régimen interior de los centros educativos, basados en la reflexión y la acción de cambio, iniciados con la gradual eliminación de las limitaciones de las prohibiciones en los centros escolares, por ejemplo.

- Ofrecer formación a las familias que enseñe a los padres cómo y cuándo introducir los teléfonos móviles en la vida de sus hijos.

- Sin una formación precisa de cómo utilizarlos, tendrán más dificultades para realizar dicha labor.

\section{CONClusiones}

Tras nuestra revisión del estado de la cuestión, llegamos a concluir que el teléfono móvil puede llegar a ser un recurso educativo que mejora notablemente los procesos de enseñanza y aprendizaje y tras realizar nuestro trabajo de investigación vemos que hay intención por parte de los docentes de incorporarlos en el aula. No podemos olvidarnos de los riesgos que esta labor de integración de un elemento 
considerado tan disruptivo en el aula supone. Para minimizar dichos riesgos, se ha pretendido plantear actuaciones para tratar de hacer aprender a los docentes las potencialidades que los teléfonos móviles tienen como una herramienta educativa. Las instituciones educativas, en todos los niveles y ámbitos, deberían esforzarse por integrar y normalizar estas potenciales herramientas educativas.

En esta labor, hemos de implicar también a las familias, ya que de ellas dependerá el uso que vengan haciendo del teléfono móvil en los hogares desde edades más tempranas, cuestión que será primordial para que su integración final en el aula sea posible de la forma más pedagógica. Así pues, el teléfono móvil puede ser una herramienta fundamental en el desarrollo de las nuevas generaciones. Mediante una educación y formación adecuadas en el uso de las Tic desde edades tempranas, será posible reducir los riesgos e incrementar las potencialidades del uso del teléfono móvil en el ámbito educativo (Gómez Hernández y Monge López, 2013).

Cierto es que los resultados de nuestro estudio indican una predisposición muy favorable respecto a la posibilidad de utilizar el teléfono móvil en el aula con fines educativos, pero algunos resultados minoritarios hacen visible la brecha digital generacional que existe hoy en día dentro de la profesión docente en nuestro país. Cualquier persona que haya decidido dedicarse a esta exigente profesión debe implicarse en su trabajo a sabiendas que requiere actualización continua

Tras el trabajo realizado, hemos alcanzado los objetivos planteados al inicio de este trabajo, pero no debemos olvidar que los resultados de esta investigación no son concluyentes, más bien suponen un punto de partida para la reflexión sobre las posibilidades de integración del teléfono móvil como recurso educativo. Como líneas futuras de investigación proponemos ampliar la muestra, así como ir más allá haciendo un estudio sobre alumnos y familias para detectar diferencias respecto a las respuestas de los docentes.

\section{REFERENCIAS BIBLIOGRÁFICAS}

Brazuelo Grund, F.; Gallego Gil, D. J. y Cacheiro González, M. L. (2016). Los docentes ante la integración educativa del teléfono móvil en el aula. RED. Revista de Educación a Distancia, 52. doi: 10.6018/red/52/6.

García Llamas, J. L.; González Galán, M. A. y Ballesteros Velázquez, B. (Eds.) (2001). Introducción a la investigación en educación (1. ${ }^{\mathrm{a}}$ ed.). Madrid: UNED.

Gil Pascual, J. A. (2015). Metodología cuantitativa en educación. Madrid: UNED.

Gómez Hernández, P. y Monge López, C. (2013). Potencialidades del teléfono móvil como recurso innovador en el aula: Una revisión teórica. Revista DIM: Didáctica, Innovación y Multimedia, 26.

González, A. (2019). El número de usuarios de Internet en España crece en 4 millones: el 93\% de la población ya está conectada (We Are Social, 2019). Recuperado de https://marketing4ecommerce.net/el-numero-de-usuarios-de-internet-en-espana-crece-en-4-millonesel-93-de-la-poblacion-ya-esta-conectada/. 
INE (2018). Encuesta sobre equipamientos y uso de las Tecnologías de la Información y Comunicación en los Hogares.

Kemp, S. (2019). Digital 2019: Global internet use acelerates. Recuperado de https://wearesocial.com/blog/2019/01/digital-2019-global-internet-use-accelerates.

Parsons, D. (2014). The future of mobile learning and implications for education and training. En M. Ally y A. Tsinakos (Eds.). Increasing access through mobile learning [E] futuro del aprendizaje móvil y sus implicaciones en la educación y la formación] (pp. 217-229). Vancouver: Commonwealth of Learning Press. Recuperado de https://www. researchgate.net/publication/270741082_The_Future_of_Mobile_Learning_and_Implications_for_Education_and_Training.

Sáez López, J. M.; Sevillano García, M. L. y Pascual-Sevillano, M. A. (2019). Aplicación del juego ubicuo con realidad aumentada en Educación Primaria. Comunicar, 61 XXVII, 71-82. https://doi.org/10.3916/C61-2019-06.

Sevillano García, M. L. y Vázquez Cano, E. (2015). Modelos de investigación en contextos ubicuos y móviles en Educación Superior. Madrid: McGraw-Hill.

Torres Diaz, J.; Infante Moro, A. y Torres Carrión, P. (2015). Mobile learning: Perspectives. International Journal of Educational Technology in Higher Education, 12 (1), 38-49. doi: $10.7238 /$ rusc.v12i1.1944.

UNESCO (2013) - UNESCO Digital Library https://unesdoc.unesco.org/ark:/48223/pf0000227146.

Vázquez-Cano, E. y Sevillano García, M. L. (2019). Dispositivos digitales móviles en educación: El aprendizaje ubicuo (2. ${ }^{\mathrm{a}}$ ed.). Narcea Ediciones.

Zapata-Ros, M. (2015). Teorías y modelos sobre el aprendizaje en entornos conectados y ubicuos. Bases para un nuevo modelo teórico a partir de una visión crítica del «conectivismo". Education in the Knowledge Society, vol. 16, 1, 69-102. Universidad de Salamanca, Salamanca, España.

\section{AgRADECIMIENTOS}

Este trabajo se ha elaborado en el marco del Proyecto I+D+I titulado: «Gamificación y aprendizaje ubicuo en Educación Primaria. Elaboración de un mapa de competencias y recursos docentes, discentes y parentales (GAUBI)» (RTI2018-099764-B-100) (MINECO/FEDER), financiado por el Fondo Europeo de Desarrollo Regional (FEDER) y el Ministerio de Economía y Competitividad de España. 
